\title{
Magnetic and superconducting phase diagram of $\mathrm{Nb} / \mathrm{Gd} / \mathrm{Nb}$ trilayers
}

\author{
Yu. N. Khaydukov, ${ }^{1,2,3}$ A. S. Vasenko, ${ }^{4}$ E. A. Kravtsov, ${ }^{5,6}$ V. V. Progliado, ${ }^{5}$ V. D. Zhaketov, ${ }^{7}$ A. Csik, ${ }^{8}$ Yu. V. Nikitenko, ${ }^{7}$ \\ A. V. Petrenko, ${ }^{7}$ T. Keller, ${ }^{1,2}$ A. A. Golubov, ${ }^{9,10}$ M. Yu. Kupriyanov, ${ }^{3}$ V. V. Ustinov, ${ }^{5,11}$ V. L. Aksenov, ${ }^{7}$ and B. Keimer ${ }^{1}$ \\ ${ }^{1}$ Max-Planck-Institut für Festkörperforschung, Heisenbergstraße 1, D-70569 Stuttgart, Germany \\ ${ }^{2}$ Max Planck Society Outstation at the Heinz, Maier-Leibnitz, Zentrum, D-85748 Garching, Germany \\ ${ }^{3}$ Skobeltsyn Institute of Nuclear Physics, Moscow State University, 119991 Moscow, Russia \\ ${ }^{4}$ National Research University Higher School of Economics, 101000 Moscow, Russia \\ ${ }^{5}$ Institute of Metal Physics, 620180 Ekaterinburg, Russia \\ ${ }^{6}$ Fundamental Education Institute, Ural Federal University, 620002 Ekaterinburg, Russia \\ ${ }^{7}$ Joint Institute for Nuclear Research, 141980 Dubna, Russia \\ ${ }^{8}$ Institute for Nuclear Research (Atomki), Hungarian Academy of Sciences, Debrecen, Hungary \\ ${ }^{9}$ Faculty of Science and Technology and MESA ${ }^{+}$Institute for Nanotechnology, University of Twente, 7500 AE Enschede, Netherlands \\ ${ }^{10}$ Moscow Institute of Physics and Technology, Dolgoprudny, 141700 Moscow, Russia \\ ${ }^{11}$ Natural Sciences Institute, Ural Federal University, 620002 Ekaterinburg, Russia
}

(Received 17 January 2018; published 13 April 2018)

\begin{abstract}
We report on a study of the structural, magnetic, and superconducting properties of $\mathrm{Nb}(25 \mathrm{~nm}) / \mathrm{Gd}\left(d_{f}\right) / \mathrm{Nb}(25 \mathrm{~nm})$ hybrid structures of a superconductor/ ferromagnet $(\mathrm{S} / \mathrm{F})$ type. The structural characterization of the samples, including careful determination of the layer thickness, was performed using neutron and x-ray scattering with the aid of depth-sensitive mass spectrometry. The magnetization of the samples was determined by superconducting quantum interference device magnetometry and polarized neutron reflectometry, and the presence of magnetic ordering for all samples down to the thinnest $\operatorname{Gd}(0.8 \mathrm{~nm})$ layer was shown. The analysis of the neutron spin asymmetry allowed us to prove the absence of magnetically dead layers in junctions with Gd interlayer thickness larger than one monolayer. The measured dependence of the superconducting transition temperature $T_{c}\left(d_{f}\right)$ has a damped oscillatory behavior with well-defined positions of the minimum at $d_{f}=3 \mathrm{~nm}$ and the following maximum at $d_{f}=4 \mathrm{~nm}$, in qualitative agreement with prior work [J. S. Jiang et al., Phys. Rev. B 54, 6119 (1996)]. We use a theoretical approach based on the Usadel equations to analyze the experimental $T_{c}\left(d_{f}\right)$ dependence. The analysis shows that the observed minimum at $d_{f}=3 \mathrm{~nm}$ can be described by the so-called zero to $\pi$ phase transitions of highly transparent S/F interfaces with a superconducting correlation length $\xi_{f} \approx 4 \mathrm{~nm}$ in $\mathrm{Gd}$. This penetration length is several times higher than for strong ferromagnets like $\mathrm{Fe}, \mathrm{Co}$, and $\mathrm{Ni}$, thus simplifying the preparation of $\mathrm{S} / \mathrm{F}$ structures with $d_{f} \sim \xi_{f}$ which are of topical interest in superconducting spintronics.
\end{abstract}

DOI: 10.1103/PhysRevB.97.144511

\section{INTRODUCTION}

Superconductor/ ferromagnet (S/F) hybrid structures are attracting great interest nowadays due to a large number of phenomena, including $\pi$ Josephson junctions, the nonmonotonous dependence of the critical temperature $T_{c}$ on the thickness of the F layer $d_{f}$, superconducting spin valves, triplet superconductivity, etc. [1-5]. This rich physics is based on the proximity effect, i.e., the penetration of superconducting correlations from the $\mathrm{S}$ into the $\mathrm{F}$ layer over a typical distance $\xi_{f}$ of the order of 1-10 nm. This leakage leads to the damped oscillatory behavior of the pairing potential in S/F multilayers. Even for the simplest system, i.e., a S/F bilayer, this effect leads to a nontrivial $T_{c}\left(d_{f}\right)$ dependence: depending on the interface transparency, the $T_{c}\left(d_{f}\right)$ function can be oscillating, reentrant for highly transparent interfaces, or monotonously decaying for interfaces with medium or low transparency [6-10]. For a larger number of S/F interfaces the behavior of the pairing potential becomes more complicated. For $d_{f}<\xi_{f}$, the pair wave function in the $\mathrm{F}$ layer changes little, and the superconducting pair potential in the adjacent $S$ layers remains the same. The phase difference between the pair potentials in the $\mathrm{S}$ layers is then absent, which is referred to as the zero phase state. On the other hand, if $d_{f} \sim \xi_{f}$, the pair wave function may cross zero at the center of the F layer with an opposite sign or $\pi$ shift of the phase of the pair potential in the adjacent $\mathrm{S}$ layers, which is called the $\pi$ phase state $[11,12]$. An increase in the $\mathrm{F}$ layer thickness $d_{f}$ may provoke subsequent transitions from zero to $\pi$ phases or even into more complex phases $[13,14]$. The existence of the $\pi$ state leads to a number of striking phenomena. For example, the critical current in $\mathrm{S} / \mathrm{F} / \mathrm{S}$ Josephson junctions exhibits a damped oscillatory behavior with increasing $F$ layer thickness [15-19]. In the $\pi$ state the critical current is negative, and the transition from the zero to the $\pi$ state results in a sign change of the critical current. Zero to $\pi$ transitions can also be observed as density-of-states oscillations [20-23], critical temperature $T_{c}$ oscillations [24,25], or peculiarities in the electrodynamics [26] of S/F multilayers.

The S/F structures attract interest not only from the scientific point of view but also from the technological point of view as elements of superconducting spintronics [4,5,27-31]. High performance of such devices is 
predicted and realized for highly transparent $\mathrm{S} / \mathrm{F}$ interfaces with $d_{f} \sim \xi_{f}$.

One of the first $\mathrm{S} / \mathrm{F}$ systems which were proved to have high transparency were $\mathrm{Gd} / \mathrm{Nb}$ systems $[24,25]$. A series of $\mathrm{Nb} / \mathrm{Gd} / \mathrm{Nb}$ trilayers and periodic structures were prepared using magnetron sputtering, and an oscillatory $T_{c}\left(d_{f}\right)$ behavior was observed. More recently, homogeneous NbGd alloys [32] and $\mathrm{GdN} / \mathrm{Nb} / \mathrm{GdN}$ trilayers [33] were studied. However, pure gadolinium in combination with niobium has several advantages compared to other S/F systems widely used nowadays. First, gadolinium is a localized ferromagnet with a rather low (compared to $\mathrm{Fe}, \mathrm{Co}$, and $\mathrm{Ni}$ ) bulk Curie temperature of $T_{m}=293 \mathrm{~K}$ [34]. Strong localization of the magnetic moment stabilizes ferromagnetism even in ultrathin Gd layers. In contrast itinerant ferromagnets $(\mathrm{Fe}, \mathrm{Co}, \mathrm{Ni}$ ) form magnetically dead layers at the S/F interface [35-37], thus deteriorating the interface transparency. Another advantage of Gd is its ability to couple with other ferromagnets [38-42], forming nontrivial magnetic ordering patterns which can be used for the creation of superconducting spin valves [29-31]. Finally, niobium and gadolinium components are not mutually soluble in either the solid or liquid phase [43,44].

Motivated by these arguments, we prepared and thoroughly studied a series of $\mathrm{Nb}(25 \mathrm{~nm}) / \mathrm{Gd}\left(d_{f}\right) / \mathrm{Nb}(25 \mathrm{~nm})$ trilayers. Our work extends the pioneering work of Jiang et al. [25] on a set of samples whose structural and magnetic properties were thoroughly characterized by x-ray and neutron reflectometry. The resulting dependence of the superconducting $T_{c}$ on $d_{f}$ exhibits substantial quantitative differences with Ref. [25]. We discuss the origin of these differences and analyze the experimental $T_{c}\left(d_{f}\right)$ dependence in a theoretical framework based on the Usadel equations, leading to a full quantitative description of this prototypical S/F/S heterojunction system.

\section{SAMPLE FABRICATION AND EXPERIMENTAL TECHNIQUES}

The samples of the nominal structure $\mathrm{Ta}(3 \mathrm{~nm}) / \mathrm{Cu}(4 \mathrm{~nm}) /$ $\mathrm{Nb}(25 \mathrm{~nm}) / \mathrm{Gd}\left(d_{f}\right) / \mathrm{Nb}(25 \mathrm{~nm})$ (here and later SFS $x$, where $x \equiv d_{f}$ measured in nanometers) were prepared using an UHV magnetron machine ULVAC MPS-4000-C6 at constant current on $\mathrm{Al}_{2} \mathrm{O}_{3}(1 \overline{1} 02)$ substrates with a thickness of the Gd layer $d_{f}=[0.8-7.5] \mathrm{nm}$ (see inset in Fig. 1). The bilayer $\mathrm{Ta} / \mathrm{Cu}$ on the top is required to protect against oxidation and to create a neutron waveguide structure [45].

Before the deposition, the substrate was cleaned from organic contaminations with acetone and alcohol. The substrate was further cleaned in situ with reverse magnetron sputtering ( 2 min at an argon flow rate of $25 \mathrm{sccm}$ ) in the load chamber. The base pressure was lower than $2 \times 10^{-9}$ mbar. Pure argon gas (99.9998\% purity) at a flow rate of $25 \mathrm{sccm}$ was used as the sputter gas. The deposition was carried out at room temperature (about $25^{\circ} \mathrm{C}$ ) at a magnetron sputtering power of $100 \mathrm{~W}$ in an argon atmosphere of $1 \times 10^{-3}$ mbar. In these conditions $\mathrm{Nb}$, $\mathrm{Gd}, \mathrm{Cu}$, and $\mathrm{Ta}$ layers were sputtered at deposition rates of $2.35,6.85,6.45$, and $2.8 \mathrm{~nm} / \mathrm{min}$, respectively. The deposition rates were calibrated using test samples with the help of a Zygo NewView7300 white-light interferometer.

The quality of the layers and interfaces was studied by secondary neutral mass spectrometry (SNMS; SPECS GmbH Berlin INA-X type) and X-ray (XRR) and neutron reflectometries (NR). Both $\mathrm{X}$-ray and neutron reflectometries allow one to reconstruct the depth profile of $\mathrm{x}$-ray/neutron scattering length density (SLD) [46]. In addition, the SLD of neutrons is spin dependent: $\rho^{ \pm}=\rho_{0}(z) \pm c M(z)$, where the superscript denotes the sign of the neutron spin projection on the external field, $\rho_{0}(z)$ and $M(z)$ are the depth profiles of the nuclear SLD and the in-plane magnetization, and $c=0.231 \times 10^{-4} \mathrm{~nm}^{-2} / \mathrm{kG}$ is a scaling factor. Polarized neutron reflectometry (PNR) can thus be used as a depth-sensitive magnetometric method. In the part of the PNR measurements at remanence we measured the intensity of spin-flip scattering. This scattering channel allowed us to obtain information about the component of the in-plane magnetization noncollinear to the external field. We note that PNR is sensitive only to the in-plane component of the magnetization. The $\mathrm{x}$-ray reflectivity curves were measured on the PANalytical Empyrean diffractometer at the wavelength $\lambda=0.229$ $\mathrm{nm}$. In addition to PNR we used superconducting quantum interference device (SQUID) magnetometry for the magnetic measurements. The PNR experiments were conducted on the angle-dispersive reflectometer $\operatorname{NREX}(\lambda=0.428 \mathrm{~nm})$ at the research reactor FRM-II (Garching, Germany) and time-of-flight reflectometer REMUR $(\lambda=[0.15-1] \mathrm{nm})$ at the research reactor IBR-2 (Dubna, Russia). In all magnetometric
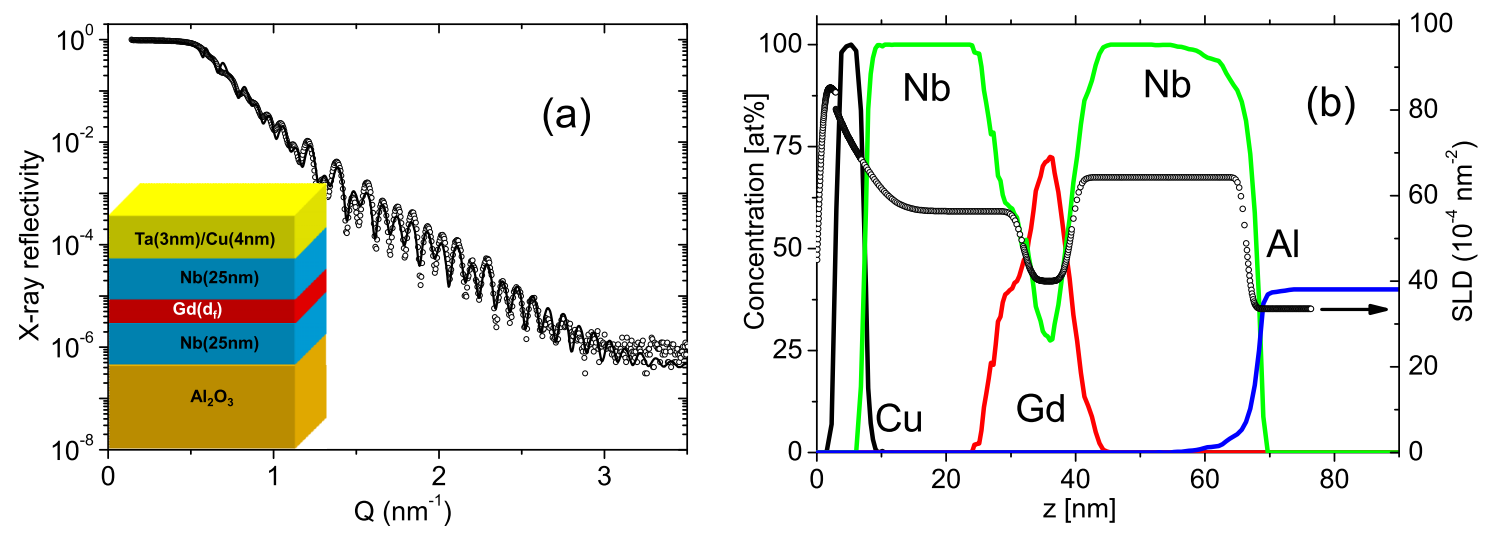

FIG. 1. (a) The x-ray experimental (dots) and theoretical (solid curves) reflectivity curves for sample SFS7.5. The inset shows the general design of the prepared structures. (b) Concentration profiles of $\mathrm{Cu}, \mathrm{Nb}, \mathrm{Gd}$, and $\mathrm{Al}$ for the same sample measured by SNMS. The dots show the $\mathrm{x}$-ray SLD depth profile for the model reflectivity depicted in (a). 
measurements, the external magnetic field was applied in the plane of the structure. Superconducting properties were measured by a SQUID magnetometer and a mutual inductance setup.

\section{STRUCTURAL PROPERTIES}

A typical $\mathrm{x}$-ray reflectivity curve measured on sample SFS7.5 is shown in Fig. 1(a). The curve exhibits so-called Kiessig oscillations caused by the interference of $\mathrm{x}$ rays reflected from different interfaces inside the structure. The experimental curve can be reasonably well fitted by the model reflectivity calculated for the SLD depth profile depicted in Fig. 1(b). In the same figure we show the concentration depth profile measured by SNMS. One can see a good correspondence between the layer thicknesses obtained with different methods. By further analysis of the depth profiles we can conclude that the real thickness of the layers deviates by at most $10 \%$ from the nominal values and that the interfaces are characterized by an $\mathrm{rms}$ roughness of the order of $1 \mathrm{~nm}$.

A similar data treatment was performed for the other samples. In the cases when SNMS was not measured, we fitted x-ray and neutron reflectivity curves simultaneously while keeping the parameters $d_{s}$ and $d_{f}$ the same for both curves. Thicknesses of all samples are within $10 \%$ of the nominal values. The rms roughness of the $\mathrm{Gd} / \mathrm{Nb}$ interfaces obtained from the fits is $0.5-1 \mathrm{~nm}$.

\section{MAGNETIC PROPERTIES}

The spin-polarized neutron reflectivities measured on sample SFS3 at $T=6.2 \mathrm{~K}$ and $H=4 \mathrm{kOe}$ are shown in Fig. 2(a). The nonzero spin asymmetry $S \equiv\left(R^{+}-R^{-}\right) /\left(R^{+}+R^{-}\right)$ [Fig. 2(b)] evidences the presence of a magnetic moment in our system. The inset in Fig. 2(a) shows the depth profiles $\rho^{+}(z)$ and $\rho^{-}(z)$ corresponding to the best-fit model. One can see that the splitting of the curves is due to the presence of a magnetization $M=7.5 \mathrm{kG}$ in the Gd layers (here and below we assume that the magnetization is already multiplied by a factor of $4 \pi$ ).

We paid particular attention to possible magnetic dead layers in our samples. First of all, we note that the sample with the thinnest $d_{f}=0.8 \mathrm{~nm}$ is still ferromagnetic, which gives us a lower bound on the thickness $d_{D L}$ of the dead layer. We also included dead layers in our models of the PNR data. In Fig. 2(b) we show the calculated spin asymmetries for three models: no magnetic dead layer (model 1), a dead layer with thickness $d_{D L}=0.5 \mathrm{~nm}$ at the bottom $\mathrm{S} / \mathrm{F}$ interface (model 2), and a dead layer at the top $\mathrm{S} / \mathrm{F}$ interface (model 3). In all models the total magnetic moment is constrained to be equal to the macroscopic moment measured by SQUID magnetometry. Model 1 provides the best description of the data with goodness of fit $\chi^{2}=8.6$. Models 2 and 3 show worse agreement with experiment with $\chi^{2}=9.8$ and $\chi^{2}=9.5$, respectively. We also tried to model the presence of dead layers on both interfaces and ended up with $\chi^{2}=9.7$.

The temperature dependence of the magnetic moment measured by SQUID in a magnetic field $H=661$ Oe on sample SFS3 is shown in Fig. 3(a). The SQUID measurements have to be carried out in low magnetic fields due to the diamagnetic

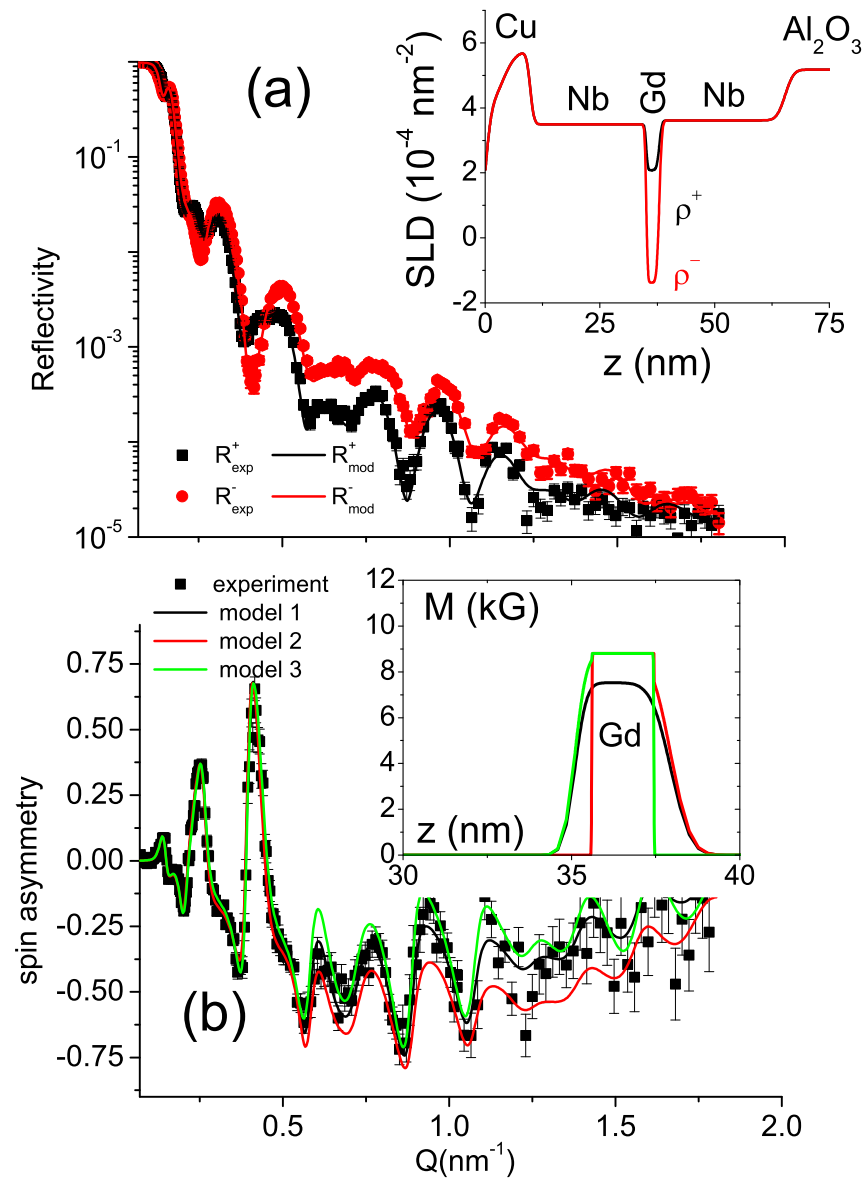

FIG. 2. (a) Experimental (dots) and model (solid line) neutron reflectivity curves for sample SFS3 measured at $T=6.2 \mathrm{~K}$ and $H=4$ kOe. The inset shows the SLD depth profiles for spin-up $\left(\rho^{+}\right)$and spin-down $\left(\rho^{-}\right)$neutrons corresponding to the best-fit model. (b) The experimental (dots) spin asymmetry for the reflectivities depicted in (a). The solid lines show the model curves for the magnetic profiles which are shown in the inset.

response of the substrate [see Fig. 3(b)]. The smaller $d_{f}$ is, the higher the field range is where the magnetic signal of the substrate dominates over the signal of the F layer. The PNR data, in contrast, are insensitive to the magnetic moment of the substrate and can be measured in fields above saturation. In Fig. 3(a) we also show the temperature dependence of the neutron spin asymmetry measured in an applied field $H=4$ kOe. We can see good agreement between neutron and SQUID data at all temperatures down to $T=60 \mathrm{~K}$. The difference at lower temperature can be ascribed to a reorientation of the easy axis which was observed in bulk Gd [47]. The Curie temperature $T_{m}$ was extracted from the temperature dependence of $m(T)$, and the resulting dependence $T_{m}\left(d_{f}\right)$ is shown in the inset of Fig. 3(a). $T_{m}$ grows with increasing $d_{f}$ up to $d_{f} \sim 3 \mathrm{~nm}$ and then saturates at the bulk value. This behavior is in qualitative agreement with previous reports [24].

The field dependence of the SQUID magnetic moment measured at $T=13 \mathrm{~K}$ on the same sample is shown in Fig. 3(b). The hysteresis loop reveals a coercivity field of $H_{c} \approx$ $500 \mathrm{Oe}$ and saturation magnetic moment of $m_{\text {sat }} \approx 50 \mu \mathrm{emu}$. Knowing from XRR and NR the Gd layer thickness and 

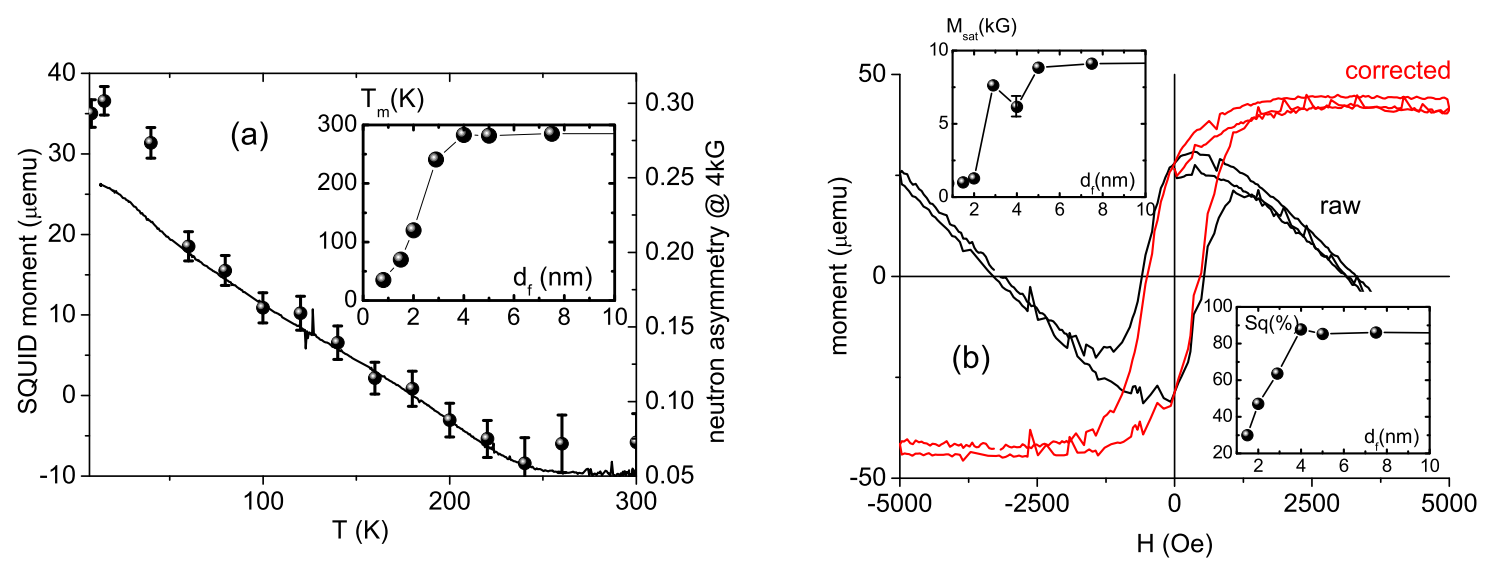

FIG. 3. (a) The temperature dependence of the SQUID magnetic moment (solid line) and averaged spin asymmetry (dots) for sample SFS3. The inset shows the dependence of $T_{m}\left(d_{f}\right)$. (b) The field dependence for sample SFS3 measured at $T=13 \mathrm{~K}$. Original and background-corrected loops are shown by black and red curves, respectively. The top and bottom insets show the $d_{f}$ dependencies of the saturation magnetization and the squareness of the hysteresis loop.

sample area $S=25 \mathrm{~mm}^{2}$ the saturation magnetization can be calculated as $M_{\text {sat }}=m_{\text {sat }} /\left(d_{f} S\right)=7.6 \mathrm{kG}$. This value is in good agreement with $M=7.5 \mathrm{kG}$ found from PNR, giving thus another cross-check of our determination of the thickness $d_{f}$ and magnetization. The top inset in Fig. 3(b) shows the dependence $M_{\text {sat }}\left(d_{f}\right)$. One can also see that $M_{\text {sat }}\left(d_{f}\right)$ correlates with $T_{m}\left(d_{f}\right)$, depicted in the inset in Fig. 3(a).

Another characteristic of a hysteresis loop is its squareness $S q \equiv m_{\text {rem }} / m_{\text {sat }}$ ( $m_{\text {rem }}$ is the remanent magnetic moment). A squareness less than $100 \%$ means that the external magnetic field was applied at an angle $\alpha \approx \arccos (S q)$ to the easy axis direction. The $S q\left(d_{f}\right)$ dependence shown in the bottom inset of Fig. 3(b) tells us that the easy axis (EA) of all samples makes a nonzero angle with $H$. If the EA lies in the plane of the structure, we should observe neutron spin-flip scattering, which, however, was not observed in the experiment. This leads us to the conclusion that the EA is aligned out of plane.

\section{SUPERCONDUCTING PROPERTIES AND THE PROXIMITY EFFECT}

The temperature dependencies of the magnetic moment around $T_{c}$ in different magnetic fields measured by SQUID on the SFS3 sample are shown in Fig. 4(a). In magnetic fields $H<0.5 \mathrm{kOe}$ the total magnetic moment decreases below $T_{c}$ due to the Meissner response of the $\mathrm{S}$ layers. For fields $H>0.5 \mathrm{kOe}$, in contrast, an increase in the magnetic moment is observed with almost linear dependence of the jump on magnetic field [inset in Fig. 4(a)]. A similar jump with linear dependence was observed recently in $(\mathrm{Fe}, \mathrm{Co}, \mathrm{Ni}) / \mathrm{V}$ bilayers [48]. It is interesting to note that PNR, in contrast, does not reveal any difference in the spin asymmetries above and below $T_{c}$ within the statistical accuracy.

The field dependence of $T_{c}$ is shown by red dots in the inset of Fig. 4(a). The $T_{c}(H)$ dependence for this sample has a linear form typical for three-dimensional superconductors which evidences coupling of the $\mathrm{S}$ layers through the $\mathrm{F}$ one. By fitting the experimental dependence $T_{c}(H)$ we can extract $T_{c}(0)=$ $5.7 \mathrm{~K}$ and $H_{c 2}(0)=12.6 \mathrm{kOe}$. The latter value allows us to estimate the superconducting correlation length $\xi_{s} \sim 10 \mathrm{~nm}$.
The $T_{c}\left(d_{f}\right)$ dependence is shown in Fig. 4(b). It has a damped oscillatory behavior with a minimum at $d_{f}=3 \mathrm{~nm}$ followed by a maximum at $d_{f}=4 \mathrm{~nm}$. The shape of the curve is similar to the one measured by Jiang et al. [25] for similar $\mathrm{Nb}(25 \mathrm{~nm}) / \mathrm{Gd} / \mathrm{Nb}(25 \mathrm{~nm})$ trilayers; however, both minimum and maximum are observed in our case at $\delta d_{f} \sim$ $1.5 \mathrm{~nm}$ higher values. After taking this offset into account, both $T_{c}\left(d_{f}\right)$ dependencies become consistent.

\section{DISCUSSION AND CONCLUSION}

In this work we have studied the $d_{f}$ dependence of the magnetic and superconducting properties of $\mathrm{Nb}(25 \mathrm{~nm}) / \mathrm{Gd}\left(d_{f}\right) / \mathrm{Nb}(25 \mathrm{~nm}) \mathrm{S} / \mathrm{F} / \mathrm{S}$ trilayers. The thickness $d_{f}$ was derived from comprehensive analysis of several structural and magnetic techniques such as secondary neutral mass spectrometry, x-ray and neutron reflectometries, and SQUID magnetometry. The magnetic and superconducting transition temperatures generally agree with the ones reported in Ref. [24] if an offset in the thickness of the Gd layer $\delta d_{f} \sim 1.5 \mathrm{~nm}$ is taken into account. The difference cannot be explained by the presence of magnetically dead layers in our structures since both PNR and SQUID data exclude the presence of any dead layer with a thickness of more than one monolayer. Taking into account the similar Curie temperatures of the $\mathrm{S} / \mathrm{F} / \mathrm{S}$ systems in this work and analogous trilayers in Ref. [25], the offset cannot be explained by different experimental conditions either. We attribute the difference to a miscalibration of the thicknesses of the $\mathrm{Gd}$ layer in Refs. [24,25]. In those works the thicknesses were calibrated using a quartz-crystal monitor and the position of the Bragg peaks in the x-ray reflectivities. However, the quartz-crystal monitor has a sensitivity of the order of $1 \mathrm{~nm}$, and the positions of the low-order Bragg peaks are shifted towards higher angles due to the refraction effect. This means that attempts to calculate the thickness of a $\mathrm{Nb} / \mathrm{Gd}$ bilayer using the standard Bragg law will generate a systematic error of 1-3 nm (see Appendix B). Taking into account this offset allows us to reconcile the dependencies of $T_{c}\left(d_{f}\right)$ in both cases. 

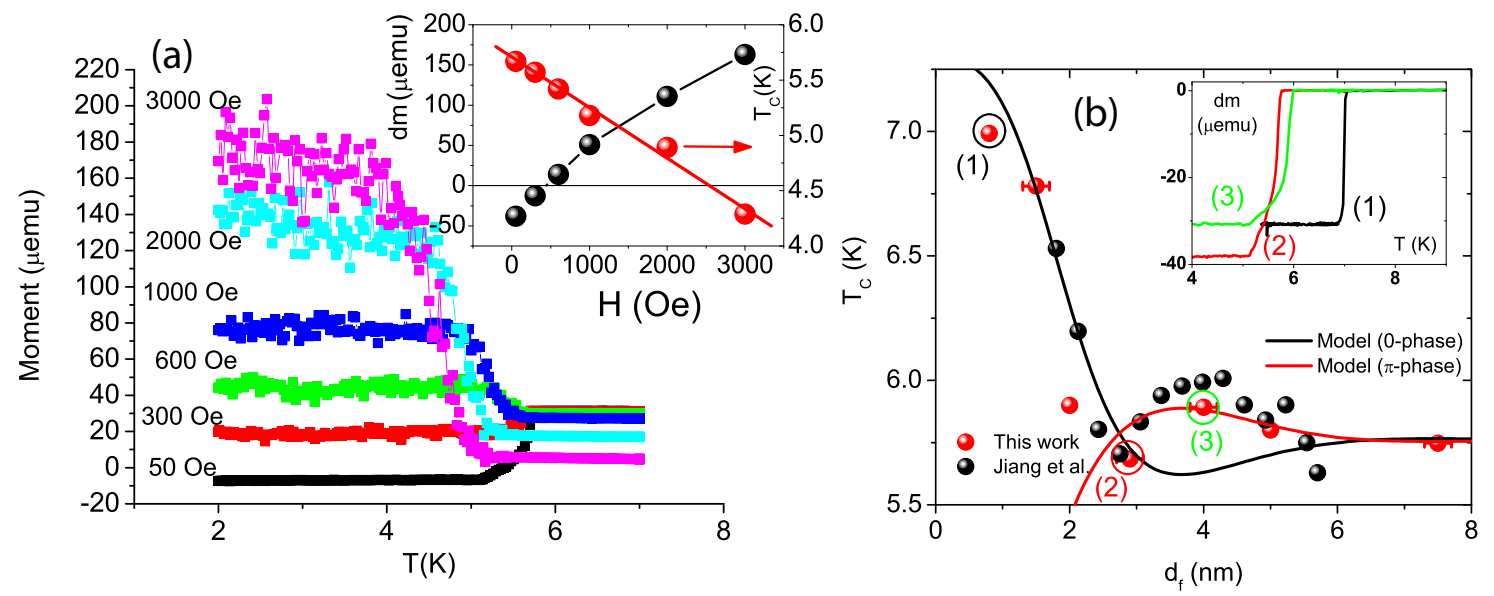

FIG. 4. (a) Temperature dependence of the magnetic moment measured by SQUID on sample SFS3. The inset shows the field dependence of the upturn amplitude and $T_{c}$ (defined as the midpoint of the transition). (b) Experimental $T_{c}\left(d_{f}\right)$ dependence for this work (red dots) and Ref. [25] (black dots) with $\delta d_{f}=1.5 \mathrm{~nm}$ correction. Black and red lines show calculations using the Usadel equations for zero and $\pi$ phase states. The inset shows the temperature dependence of the magnetic moment change $d m(T) \equiv m(T)-m\left(T>T_{c}\right)$ for the set of samples marked in the main panel.

We compared the experimental $T_{c}\left(d_{f}\right)$ dependencies to model curves calculated for the zero and $\pi$ states using the Usadel approach (see Appendix A). For the calculations we fixed $\xi_{s}=10 \mathrm{~nm}$ and the exchange energy $E_{e x}=280 \mathrm{~K}$ and varied $\xi_{f}, \gamma$, and $\gamma_{b}$. The parameters $\gamma$ and $\gamma_{b}$ are expressed via the normal-state conductivity of the $\mathrm{S}(\mathrm{F})$ layer $\sigma_{s(n)}$, the resistance of the S/F boundary $R_{B}$, and abovedefined correlation lengths as $\gamma=\xi_{s} \sigma_{n} / \xi_{n} \sigma_{s}, \gamma_{B}=R_{B} \sigma_{n} / \xi_{n}$. Reasonably good agreement between experiment and theory was obtained for $\gamma=0.07, \gamma_{b} \rightarrow 0$, and $\xi_{f}=4 \mathrm{~nm}$. The extremely small parameter $\gamma_{b}$ indicates a high transparency of the S/F interface. Thus, according to our calculations the superconducting correlations penetrate into the Gd layer with a typical length of $\xi_{f}=4 \mathrm{~nm}$. The $\pi$ state becomes energetically favorable for a region of thickness $d_{f}=$ [3-6] nm. For higher thicknesses transmission of the correlations through the F layer becomes impossible, and the S/F/S structure splits into two independent $\mathrm{S} / \mathrm{F}$ bilayers.

We now discuss the magnetic properties of the samples. Our investigation has shown that samples with $d_{f}>2 \mathrm{~nm}$ have Curie temperatures close to the bulk and almost square hysteresis loops. However, the magnetic moment of our structures is only $3.7 \mu_{B} / \mathrm{Gd}$, i.e., roughly half of the bulk value. A similarly suppressed moment was found in $\mathrm{Gd} / \mathrm{U}$ [49], $\mathrm{Gd} / \mathrm{V}$ [50], and $\mathrm{Gd} / \mathrm{Cr}$ [51] multilayers. This suppression may well be related to the presence of the less magnetic fcc phase together with the bulk hcp phase which was recently found in $\mathrm{Fe} / \mathrm{Cr} / \mathrm{Gd}$ multilayers [40].

Below $T_{c}$ we observed an upturn of the magnetic moment if the sample was cooled down in a certain magnetic field. A similar upturn, often called the paramagnetic Meissner effect (PME), was already observed in several prior works [48,52-56] and was explained by either electrodynamic or exchange coupling mechanisms. Based on (a) the observation of the effect at high fields, (b) the linear field dependence of the enhanced moment, and (c) the absence of the effect in PNR, we attribute the PME in our samples to out-of-plane vortices. In Ref. [57] the PME for a single S film with the external field directed normal to the surface was explained by vortex trapping. In our case the stray field of $\mathrm{Gd}$ can play the role of the out-of-plane external field. It is also known that the proximity effect can influence the vortex dynamics and hence cause a PME [58]. This question has to be addressed by future investigations.

In conclusion, we have shown that high-quality $\mathrm{Nb} / \mathrm{Gd} / \mathrm{Nb}$ trilayers can be grown using magnetron sputtering in a wide range of thicknesses. The penetration depth of superconducting correlations in the Gd layer is found to be several times higher than for strong ferromagnets like $\mathrm{Fe}, \mathrm{Co}$, or Ni. This simplifies the preparation of S/F structures with $d_{f} \sim \xi_{f}$ which are of topical interest in superconducting spintronics.

\section{ACKNOWLEDGMENTS}

The authors would like to thank G. Nowak and V. Zdravkov for fruitful discussions and R. Morari for assistance with the experiment. This work is partially based on experiments performed at the NREX instrument operated by the Max Planck Society at the Heinz Maier-Leibnitz Zentrum (MLZ), Garching, Germany, and supported by DFG collaborative research center TRR 80. A.A.G, M.Yu.K., and A.S.V. acknowledge support from joint Russian-Greek projects RFMEFI61717X0001 and T4P-37200031 "Experimental and theoretical studies of physical properties of lowdimensional quantum nanoelectronic systems." Research in Ekaterinburg was performed in terms of the state assignment of the Federal Agency of Scientific Organizations of the RF (theme "Spin" No. AAAA-A18-188020290104-2) and according to Contract No. 14.Z50.31.0025 with the Ministry of Science and Education of the RF. The x-ray measurements were performed at the Collective Use Center of the IMP. SNMS measurements were carried out in the frame of the GINOP-2.3.2-15-2016-00041 project, which is cofinanced by the European Union and the European Regional Development Fund. 


\section{APPENDIX A: CRITICAL-TEMPERATURE CALCULATION}

The model of the $\mathrm{S} / \mathrm{F} / \mathrm{S}$ junction we are going to study is depicted in Fig. 5 and consists of a ferromagnetic layer of thickness $d_{f}$ and two superconducting layers of thickness $d_{s}$ along the $x$ direction. The structure is symmetric, and its center is placed at $x=0$. We assume the diffusive limit and $\hbar=k_{B}=1$.

To calculate the critical temperature $T_{c}\left(d_{f}\right)$ of this structure we use the framework of the linearized Usadel equations for the $\mathrm{S}$ and F layers. Near $T_{c}$ the normal Green's function is $G=$ $\operatorname{sgn} \omega_{n}$, and the Usadel equations for the anomalous Green's function $F$ in the $\mathrm{S}$ layers reads $\left(d_{f} / 2<|x|<d_{s}+d_{f} / 2\right)$ [59]

$$
\xi_{s}^{2} \pi T_{c s} \frac{d^{2} F_{s}}{d x^{2}}-\left|\omega_{n}\right| F_{s}+\Delta=0 .
$$

In the $\mathrm{F}$ layer $\left(-d_{f} / 2<x<d_{f} / 2\right)$ the Usadel equation can be written as [1]

$$
\xi_{n}^{2} \pi T_{c s} \frac{d^{2} F_{f}}{d x^{2}}-\left(\left|\omega_{n}\right|+i E_{e x} \operatorname{sgn} \omega_{n}\right) F_{f}=0 .
$$

Finally, the self-consistency equation reads [1],

$$
\Delta \ln \frac{T_{c s}}{T}=\pi T \sum_{\omega_{n}}\left(\frac{\Delta}{\left|\omega_{n}\right|}-F_{s}\right) .
$$

Here $\xi_{s}=\sqrt{D_{s} / 2 \pi T_{c s}}, \xi_{n}=\sqrt{D_{f} / 2 \pi T_{c s}}, \omega_{n}=2 \pi T(n+$ $\frac{1}{2}$ ), with $n=0, \pm 1, \pm 2, \ldots$, are the Matsubara frequencies; $E_{e x}$ is the exchange field in the ferromagnet; $T_{c s}$ is the critical temperature of the $\mathrm{S}$ material $\left(d_{f} \rightarrow 0\right)$; and $F_{s(f)}$ denotes the anomalous Green's function in the $\mathrm{S}(\mathrm{F})$ region. We note that $\xi_{f}=\xi_{n} \sqrt{2 \pi T_{c s} / E_{e x}}$.

Equations (A1)-(A3) must be supplemented by the following boundary conditions at the S/F interfaces $\left(x= \pm d_{f} / 2\right)$ [60]:

$$
\begin{aligned}
\xi_{s} \frac{d F_{s}\left( \pm d_{f} / 2\right)}{d x} & =\gamma \xi_{f} \frac{d F_{f}\left( \pm d_{f} / 2\right)}{d x}, \\
\xi_{f} \gamma_{b} \frac{d F_{f}\left( \pm d_{f} / 2\right)}{d x} & = \pm F_{s}\left( \pm d_{f} / 2\right) \mp F_{f}\left( \pm d_{f} / 2\right),
\end{aligned}
$$

where $\gamma=\xi_{s} \sigma_{n} / \xi_{n} \sigma_{s}, \sigma_{s(n)}$ is the normal-state conductivity of the $\mathrm{S}(\mathrm{F})$ layer, $\gamma_{B}=R_{B} \sigma_{n} / \xi_{n}[60-62]$, and $R_{B}$ is the resistance of the $\mathrm{S} / \mathrm{F}$ interfaces (we assume a symmetric structure with

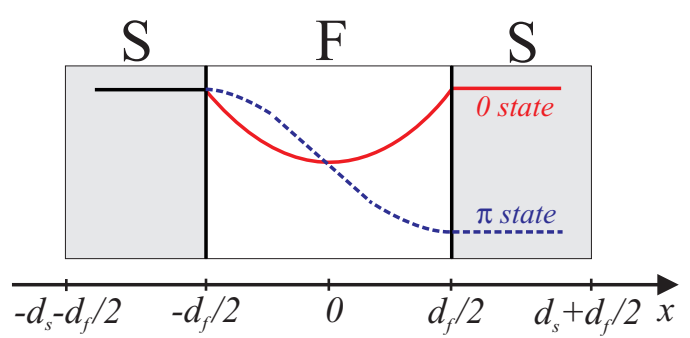

FIG. 5. Geometry of the considered system. The thickness of the ferromagnetic interlayer is $d_{f}$. The typical behavior of the real part of the pair wave function $F$ is shown schematically. The pair wave function in the zero state is shown by the solid red line, while in that the $\pi$ state is shown by dashed blue line. Only one of these states is realized depending on the F layer thickness $d_{f}$.

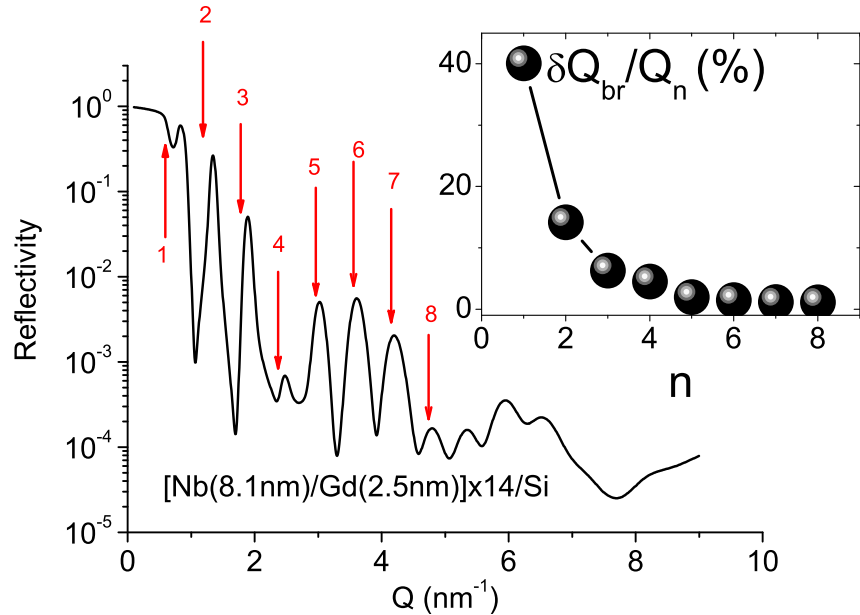

FIG. 6. Model x-ray reflectivity curve calculated for the $[\mathrm{Nb}(8.1 \mathrm{~nm}) / \mathrm{Gd}(2.5 \mathrm{~nm})] \times 14 / \mathrm{Si}$ system (compare with Fig. 1 in [24]). The vertical red arrows show the positions of the Bragg reflections calculated by Eq. (B1). The inset shows the deviation of the peak position from the Bragg law as a function of peak order.

the same resistance $R_{B}$ for $x= \pm d_{f} / 2$ ). At the borders of the $\mathrm{S}$ layer with a vacuum we naturally have

$$
\frac{d F_{s}\left( \pm d_{s} \pm d_{f} / 2\right)}{d x}=0 .
$$

The solution of the Usadel equation in the F layer depends on the phase state of the structure. In the zero phase state the anomalous Green's function is symmetric relative to $x=0$ (see Fig. 5) [6],

$$
\begin{aligned}
& F_{f}^{0}=C\left(\omega_{n}\right) \cosh \left(k_{f} x\right), \\
& k_{f}=\frac{1}{\xi_{n}} \sqrt{\frac{\left|\omega_{n}\right|+i E_{e x} \operatorname{sgn} \omega_{n}}{\pi T_{c s}} .}
\end{aligned}
$$

In the $\pi$ phase state the anomalous Green's function is antisymmetric relative to $x=0$ (see Fig. 5),

$$
F_{f}^{\pi}=C^{\prime}\left(\omega_{n}\right) \sinh \left(k_{f} x\right) .
$$

In Eqs. (A6) and (A7) $C\left(\omega_{n}\right)$ and $C^{\prime}\left(\omega_{n}\right)$ are the integration constants to be found from the boundary conditions.

The boundary value problem (A1)-(A5) can be solved in order to obtain the closed boundary condition for the $F_{s}$ function. At the right $\mathrm{S} / \mathrm{F}$ interface $\left(x=d_{f} / 2\right)$ it acquires the form

$$
\xi_{s} \frac{d F_{s}\left(d_{f} / 2\right)}{d x}=\frac{\gamma}{\gamma_{B}+B_{f}\left(\omega_{n}\right)} F_{s}\left(d_{f} / 2\right) .
$$

A similar boundary condition can be written at $x=-d_{f} / 2$. In Eq. (A8) the $B_{f}$ function can acquire different values in zero and $\pi$ phase states. The zero state was already considered in Ref. [6],

$$
B_{f}^{0}=\left[k_{f} \xi_{n} \tanh \left(k_{f} d_{f} / 2\right)\right]^{-1},
$$

while in the $\pi$ state from Eq. (A7) we obtain

$$
B_{f}^{\pi}=\left[k_{f} \xi_{n} \operatorname{coth}\left(k_{f} d_{f} / 2\right)\right]^{-1} .
$$


The boundary condition (A8) is complex. In order to rewrite it in a real form, we use the following relation:

$$
F^{ \pm}=F\left(\omega_{n}\right) \pm F\left(-\omega_{n}\right) .
$$

According to the Usadel equations (A1)-(A3), there is a symmetry relation $F\left(-\omega_{n}\right)=F^{*}\left(\omega_{n}\right)$ which implies that $F^{+}$ is real while $F^{-}$is a purely imaginary function.

Thus, we can consider only positive Matsubara frequencies and express the self-consistency equation (A3) only via the symmetric function $F^{+}$,

$$
\Delta \ln \frac{T_{c s}}{T}=\pi T \sum_{\omega_{n}>0}\left(\frac{2 \Delta}{\omega_{n}}-F_{s}^{+}\right) .
$$

The problem of $T_{c}$ determination can be formulated in a closed form with respect to $F_{s}^{+}$. Using the boundary condition (A8), we arrive at the effective boundary conditions for $F_{s}^{+}$at the right $\mathrm{S}$ layer boundaries,

$$
\begin{aligned}
\xi_{s} \frac{d F_{s}^{+}\left(d_{f} / 2\right)}{d x} & =W\left(\omega_{n}\right) F_{s}^{+}\left(d_{f} / 2\right), \\
\frac{d F_{s}^{+}\left(d_{s}+d_{f} / 2\right)}{d x} & =0 .
\end{aligned}
$$

Similar boundary conditions can be written at the left $\mathrm{S}$ layer boundaries. In Eqs. (A13) we used the notations

$$
\begin{aligned}
W^{0, \pi}\left(\omega_{n}\right) & =\gamma \frac{A_{s}\left(\gamma_{B}+\operatorname{Re} B_{f}^{0, \pi}\right)+\gamma}{A_{s}\left|\gamma_{B}+B_{f}^{0, \pi}\right|^{2}+\gamma\left(\gamma_{B}+\operatorname{Re} B_{f}^{0, \pi}\right)}, \\
A_{s} & =k_{s} \xi_{s} \tanh \left(k_{s} d_{s}\right), \quad k_{s}=\frac{1}{\xi_{s}} \sqrt{\frac{\omega_{n}}{\pi T_{c s}}} .
\end{aligned}
$$

The self-consistency equation (A12) and boundary conditions (A13), together with the Usadel equation for $F_{s}^{+}$,

$$
\xi_{s}^{2} \pi T_{c s} \frac{d^{2} F_{s}^{+}}{d x^{2}}-\omega_{n} F_{s}^{+}+2 \Delta=0,
$$

can be used to find the critical temperature of the $S / F / S$ structure both in zero and $\pi$ phase states. In general, this problem should be solved numerically [6]. In Ref. [6] it was also found that the so-called single-mode approximation (SMA) essentially simplifies the numerical problems and gives the $T_{c}\left(d_{f}\right)$ dependency with an accuracy which is high enough for our consideration. In the SMA the self-consistency equation (A12) takes the form [6]

$$
\ln \frac{T_{c s}}{T_{c}}=\psi\left(\frac{1}{2}+\frac{\Omega^{2}}{2} \frac{T_{c s}}{T_{c}}\right)-\psi\left(\frac{1}{2}\right),
$$

where $\psi$ is the digamma function and $\Omega$ can be found from the following equation:

$$
\Omega \tan \left(\Omega \frac{d_{s}}{\xi_{s}}\right)=W^{0, \pi}\left(\omega_{n}\right) .
$$

The critical temperature $T_{c}\left(d_{f}\right)$ is then determined by Eqs. (A16) and (A17). This result extends the result of Ref. [6] to the case of $\mathrm{S} / \mathrm{F} / \mathrm{S}$ hybrid structures, where the $\pi$ phase state can be realized for large enough $\mathrm{F}$ layer thickness, $d_{f} \sim \xi_{f}$. A previous approach by Buzdin and Kupriyanov [63] and Buzdin et al. [11] was formulated for thin S layers $\left(d_{s} / \xi_{s} \ll 1\right)$ and in the limit of small critical-temperature variations $\left(T_{c s}-T_{c}\right) / T_{c s} \ll 1$, while calculations done in [6] were focused only on large $\mathrm{F}$ layer thickness.

\section{APPENDIX B: X-RAY REFLECTOMETRY FOR THE DETERMINATION OF THE LAYER THICKNESSES IN PERIODIC STRUCTURES}

The position of Bragg reflections in diffraction experiments from structures with periodicity $D$ can be written as

$$
Q_{B r}=2 \pi n / D,
$$

where $n$ is an integer. Reflectometric experiments also show Bragg-like peaks, although their positions deviate from Bragg's law close to the total external reflection due to the refraction effect [64]. As an example we show in Fig. 6 the calculated $x$-ray reflectivity curve for the structure $[\mathrm{Nb}(8.1 \mathrm{~nm}) 7 \mathrm{sol} ; \mathrm{Gd}(2.5 \mathrm{~nm})] \times 14 / \mathrm{Si}$ described in [24]. Vertical arrows correspond to the Bragg law equation (B1). One can see that the positions of the maxima are shifted towards higher values compared to the Bragg condition (B1). The deviation from the Bragg law is especially strong for smaller orders and vanishes gradually for the higher orders. Attempts to calculate the period using Eq. (B1) for the $n=1$ and $n=2$ peaks give $D=7.5 \mathrm{~nm}$ and $D=9.3 \mathrm{~nm}$, which are 3.1 and $1.3 \mathrm{~nm}$ smaller than the real thickness.
[1] A. I. Buzdin, Rev. Mod. Phys. 77, 935 (2005).

[2] A. A. Golubov, M. Y. Kupriyanov, and E. Il'ichev, Rev. Mod. Phys. 76, 411 (2004).

[3] F. S. Bergeret, A. F. Volkov, and K. B. Efetov, Rev. Mod. Phys. 77, 1321 (2005).

[4] M. Eschrig, Phys. Today 64(1), 43 (2011).

[5] A. Sidorenko, Low Temp. Phys. 43, 766 (2017).

[6] Y. V. Fominov, N. M. Chtchelkatchev, and A. A. Golubov, Phys. Rev. B 66, 014507 (2002).

[7] V. Zdravkov, A. Sidorenko, G. Obermeier, S. Gsell, M. Schreck, C. Müller, S. Horn, R. Tidecks, and L. R. Tagirov, Phys. Rev. Lett. 97, 057004 (2006).

[8] V. I. Zdravkov, J. Kehrle, G. Obermeier, S. Gsell, M. Schreck, C. Müller, H.-A. Krug von Nidda, J. Lindner, J. Moosburger-Will,
E. Nold, R. Morari, V. V. Ryazanov, A. S. Sidorenko, S. Horn, R. Tidecks, and L. R. Tagirov, Phys. Rev. B 82, 054517 (2010).

[9] C. Cirillo, S. L. Prischepa, M. Salvato, C. Attanasio, M. Hesselberth, and J. Aarts, Phys. Rev. B 72, 144511 (2005).

[10] A. A. Armenio, C. Cirillo, G. Iannone, S. L. Prischepa, and C. Attanasio, Phys. Rev. B 76, 024515 (2007).

[11] A. Buzdin, B. Vujicic, and M. Yu. Kupriyanov, Zh. Eksp. Teor. Phys. 101, 231 (1992) [Sov. Phys. JETP 74, 124 (1992)].

[12] E. A. Demler, G. B. Arnold, and M. R. Beasley, Phys. Rev. B 55, 15174 (1997).

[13] V. N. Kushnir, S. L. Prischepa, C. Cirillo, A. Vecchione, C. Attanasio, M. Y. Kupriyanov, and J. Aarts, Phys. Rev. B 84, 214512 (2011)

[14] V. N. Kushnir and M. Y. Kupriyanov, JETP Lett. 93, 539 (2011). 
[15] A. I. Buzdin and M. Yu. Kupriyanov, Pis'ma Zh. Eksp. Teor. Fiz. 53, 308 (1991) [JETP Lett. 53, 321 (1991)].

[16] V. V. Ryazanov, V. A. Oboznov, A. Y. Rusanov, A. V. Veretennikov, A. A. Golubov, and J. Aarts, Phys. Rev. Lett. 86, 2427 (2001).

[17] V. A. Oboznov, V. V. Bol'ginov, A. K. Feofanov, V. V. Ryazanov, and A. I. Buzdin, Phys. Rev. Lett. 96, 197003 (2006).

[18] M. Weides, M. Kemmler, E. Goldobin, D. Koelle, R. Kleiner, H. Kohlstedt, and A. Buzdin, Appl. Phys. Lett. 89, 122511 (2006).

[19] M. Kemmler, M. Weides, M. Weiler, M. Opel, S. T. B. Goennenwein, A. S. Vasenko, A. A. Golubov, H. Kohlstedt, D. Koelle, R. Kleiner, and E. Goldobin, Phys. Rev. B 81, 054522 (2010).

[20] T. Kontos, M. Aprili, J. Lesueur, and X. Grison, Phys. Rev. Lett. 86, 304 (2001).

[21] A. Buzdin, Phys. Rev. B 62, 11377 (2000).

[22] A. S. Vasenko, A. A. Golubov, M. Y. Kupriyanov, and M. Weides, Phys. Rev. B 77, 134507 (2008).

[23] A. S. Vasenko, S. Kawabata, A. A. Golubov, M. Y. Kupriyanov, C. Lacroix, F. S. Bergeret, and F. W. J. Hekking, Phys. Rev. B 84, 024524 (2011).

[24] J. S. Jiang, D. Davidović, D. H. Reich, and C. L. Chien, Phys. Rev. Lett. 74, 314 (1995).

[25] J. S. Jiang, D. Davidović, D. H. Reich, and C. L. Chien, Phys. Rev. B 54, 6119 (1996).

[26] N. Pompeo, K. Torokhtii, C. Cirillo, A. V. Samokhvalov, E. A. Ilyina, C. Attanasio, A. I. Buzdin, and E. Silva, Phys. Rev. B 90, 064510 (2014).

[27] I. I. Soloviev, N. V. Klenov, S. V. Bakurskiy, M. Y. Kupriyanov, A. L. Gudkov, and A. S. Sidorenko, Beilstein J. Nanotechnol. 8, 2689 (2017).

[28] A. A. Golubov and M. Y. Kupriyanov, Nat. Mater. 16, 156 (2017).

[29] A. Singh, S. Voltan, K. Lahabi, and J. Aarts, Phys. Rev. X 5, 021019 (2015).

[30] Y. V. Fominov, A. A. Golubov, T. Y. Karminskaya, M. Y. Kupriyanov, R. G. Deminov, and L. R. Tagirov, JETP Lett. 91, 308 (2010).

[31] L. R. Tagirov, Phys. Rev. Lett. 83, 2058 (1999).

[32] A. Bawa, A. Gupta, S. Singh, V. Awana, and S. Sahoo, Sci. Rep. 6, 18689 (2016).

[33] Y. Zhu, A. Pal, M. G. Blamire, and Z. H. Barber, Nat. Mater. 16, 195 (2016).

[34] W. C. Koehler, J. of Appl. Phys. 36, 1078 (1965).

[35] T. Mühge, K. Westerholt, H. Zabel, N. N. Garif'yanov, Y. V. Goryunov, I. A. Garifullin, and G. G. Khaliullin, Phys. Rev. B 55, 8945 (1997).

[36] Y. Obi, M. Ikebe, T. Kubo, and H. Fujimori, Phys. C (Amsterdam, Neth.) 317-318, 149 (1999).

[37] Y. Obi, M. Ikebe, and H. Fujishiro, Phys. Rev. Lett. 94, 057008 (2005).

[38] Y. Choi, D. Haskel, R. E. Camley, D. R. Lee, J. C. Lang, G. Srajer, J. S. Jiang, and S. D. Bader, Phys. Rev. B 70, 134420 (2004).

[39] E. Kravtsov, D. Haskel, S. G. E. te Velthuis, J. S. Jiang, and B. J. Kirby, Phys. Rev. B 79, 134438 (2009).

[40] M. V. Ryabukhina, E. A. Kravtsov, D. V. Blagodatkov, L. I. Naumova, Y. V. Nikitenko, V. V. Proglyado, and Y. N. Khaydukov, J. Surf. Invest.: X-ray, Synchrotron Neutron Tech. 9, 41 (2015).
[41] B. Sanyal, C. Antoniak, T. Burkert, B. Krumme, A. Warland, F. Stromberg, C. Praetorius, K. Fauth, H. Wende, and O. Eriksson, Phys. Rev. Lett. 104, 156402 (2010).

[42] T. Higgs, S. Bonetti, H. Ohldag, N. Banerjee, X. Wang, A. Rosenberg, Z. Cai, J. Zhao, K. Moler, and J. Robinson, Sci. Rep. 6, 30092 (2016).

[43] R. Elliot, Constitution of Binary Alloys (McGraw-Hill, New York, 1965), p. 256.

[44] F. Shunk, Constitution of Binary Alloys (McGraw-Hill, New York, 1969), p. 184.

[45] Y. Khaydukov, E. Kravtsov, V. Progliado, V. Ustinov, Y. Nikitenko, T. Keller, V. Aksenov, and B. Keimer, J. Phys.: Conf. Ser. 746, 012064 (2016).

[46] Edited by J. Daillant and A. Gibaud, X-ray and Neutron Reflectivity: Principles and Applications, Lecture Notes in Physics Vol. 770 (Springer, Berlin, Heidelberg, 2009).

[47] S. Y. Dan'kov, A. M. Tishin, V. K. Pecharsky, and K. A. Gschneidner, Phys. Rev. B 57, 3478 (1998).

[48] B. Nagy, Y. Khaydukov, D. Efremov, A. Vasenko, L. Mustafa, J.-H. Kim, T. Keller, K. Zhernenkov, A. Devishvili, R. Steitz et al., Europhys. Lett. 116, 17005 (2016).

[49] R. Springell, S. Langridge, A. Wildes, S. B. Wilkins, C. SanchezHanke, K. T. Moore, M. T. Butterfield, J. Chivall, R. C. C. Ward, M. R. Wells, and G. H. Lander, Phys. Rev. B 81, 134434 (2010).

[50] P. Pankowski, L. T. Baczewski, T. Story, A. Wawro, K. Mergia, and S. Messoloras, Phys. Status Solidi C 1, 405 (2004).

[51] K. Mergia, L. Baczewski, S. Messoloras, S. Hamada, T. Shinjo, H. Gamari-Seale, and J. Hauschild, Appl. Phys. A 74, s1520 (2002).

[52] D. K. Satapathy, M. A. Uribe-Laverde, I. Marozau, V. K. Malik, S. Das, T. Wagner, C. Marcelot, J. Stahn, S. Brück, A. Rühm, S. Macke, T. Tietze, E. Goering, A. Frañó, J. H. Kim, M. Wu, E. Benckiser, B. Keimer, A. Devishvili, B. P. Toperverg, M. Merz, P. Nagel, S. Schuppler, and C. Bernhard, Phys. Rev. Lett. 108, 197201 (2012).

[53] A. Di Bernardo, Z. Salman, X. L. Wang, M. Amado, M. Egilmez, M. G. Flokstra, A. Suter, S. L. Lee, J. H. Zhao, T. Prokscha, E. Morenzoni, M. G. Blamire, J. Linder, and J. W. A. Robinson, Phys. Rev. X 5, 041021 (2015).

[54] M. A. López de la Torre, V. Peña, Z. Sefrioui, D. Arias, C. Leon, J. Santamaria, and J. L. Martinez, Phys. Rev. B 73, 052503 (2006).

[55] C. Monton, F. de la Cruz, and J. Guimpel, Phys. Rev. B 77, 104521 (2008).

[56] G. Ovsyannikov, V. Demidov, Y. N. Khaydukov, L. Mustafa, K. Constantinian, A. Kalabukhov, and D. Winkler, J. Exp. Theor. Phys. 122, 738 (2016).

[57] A. E. Koshelev and A. I. Larkin, Phys. Rev. B 52, 13559 (1995).

[58] A. A. Golubov, M. Y. Kupriyanov, and M. M. Khapaev, JETP Lett. 104, 847 (2016).

[59] K. D. Usadel, Phys. Rev. Lett. 25, 507 (1970).

[60] M. Yu. Kuprianov and V. F. Lukichev, Zh. Eksp. Teor. Fiz. 94, 139 (1988) [Sov. Phys. JETP 67, 1163 (1988)].

[61] E. V. Bezuglyi, A. S. Vasenko, V. S. Shumeiko, and G. Wendin, Phys. Rev. B 72, 014501 (2005).

[62] E. V. Bezuglyi, A. S. Vasenko, E. N. Bratus, V. S. Shumeiko, and G. Wendin, Phys. Rev. B 73, 220506 (2006).

[63] A. I. Buzdin and M. Yu. Kupriyanov, JETP Lett. 52, 487 (1990).

[64] M. A. Andreeva and B. Lindgren, Phys. Rev. B 72, 125422 (2005). 